\title{
Improved Treatment Acceptance and Adherence Following the Diagnosis of Multiple Malformations of Cortical Development in a Patient with Psychosis
}

Rachit Patel*, Kathleen M Stuart and Draupathi Nambudiri

Department of Psychiatry, Stamford Hospital, Stamford, Connecticut, USA

\begin{abstract}
The increased use of neuroimaging has revealed a variety of malformations of cortical development (MCDs) presenting with a range of neuropsychiatric disorders, including psychotic illnesses. Non-adherence with antipsychotic medication is a common barrier to the effective treatment for psychosis. This case illustrates improved treatment acceptance and adherence following the diagnosis of two separate MCDs (bilateral periventricular heterotopia and focal cortical dysplasia) in a 48-year-old Caucasian male with psychosis. By incorporating the neuroimaging findings into a cognitive behavioral therapy approach, the patient was more amenable to accepting psychotropic medications including long-acting risperidone injection. This in turn led to an improvement in his overall functioning Furthermore, this case adds to the literature by describing the first instance of psychotic symptoms occurring in the setting of both bilateral periventricular heterotopia and focal cortical dysplasia.
\end{abstract}

\section{Keywords: Cortical development; Psychosis; Neurological disorder}

Objective: Report a case demonstrating improved treatment acceptance and adherence following the diagnosis of multiple malformations of cortical development in a patient with psychosis.

\section{Background}

Malformations of cortical development (MCDs) are macroscopic or microscopic abnormalities of the cerebral cortex that arise as a consequence of an interruption to the normal steps of formation of the cortical plate [1]. The increased use of neuroimaging has revealed a variety of MCDs presenting with a range of neuropsychiatric disorders, including psychotic illnesses [2-4]. Non-adherence with antipsychotic medication is a common barrier to the effective treatment for psychosis [5]. This case illustrates improved acceptance of and adherence to treatment following the diagnosis of two separate MCDs (bilateral periventricular heterotopia and focal cortical dysplasia) in a patient with psychosis.

\section{Case Report}

A 48-year-old Caucasian male with a five year history of psychotic illness resulting in three prior inpatient psychiatric hospitalizations was admitted after presenting with worsening paranoid delusions and referential thinking in the setting of medication non-adherence. The patient demonstrated poor insight into his illness as well as the need for treatment despite the impact on his social and occupational functioning. The patient was started on risperidone $2 \mathrm{mg} / \mathrm{day}$, which he was initially reluctant to accept despite psychoeducation about his illness. Routine laboratory studies and toxicology were negative except for vitamin B12 deficiency with a level of 226 (normal 239-931), which was treated with cyanocobalamin 1,000 mcg IM daily. CT Head revealed bilateral nodular periventricular heterotopia (Figure 1). MRI Brain further revealed a focal cortical dysplasia in the right frontal lobe. By incorporating the neuroimaging findings into a cognitive behavioral therapy approach, the patient was more amenable to accepting psychotropic medications including long-acting risperidone injection. With improved treatment acceptance, risperidone was titrated up to $4 \mathrm{mg} /$ day and he received $25 \mathrm{mg}$ of long-acting risperidone injection with an improvement in his psychosis. At 8-week follow-up the patient was still adherent to treatment with a complete resolution of psychotic symptoms. With an improvement in his functioning, the

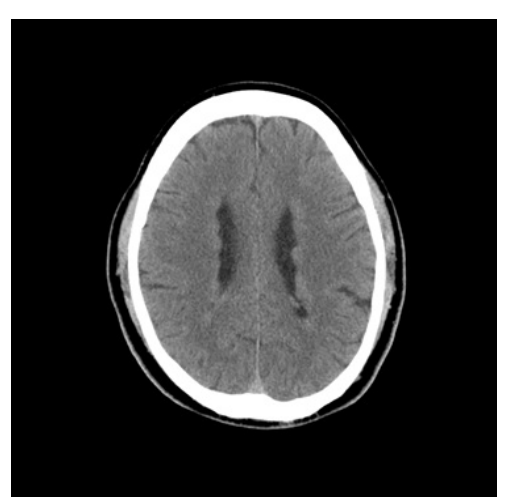

Figure 1: CT Head without contrast showing nodular contours in the margins of both lateral ventricles compatible with bilateral nodular periventricular heterotopia.

patient reported better familial relationships and was in the process of resuming his previous employment.

\section{Discussion}

Treatment acceptance and adherence are major issues in the management of patients with psychotic illnesses. It is well established that antipsychotic medications ameliorate the symptoms of psychosis. However, many patients fail to comply with drug treatment due to a lack of insight into their illness leading to an exacerbation of their symptomatology. Even with the availability of long-acting injectable medications, drug treatments alone are not enough to prevent relapse. Psychosocial interventions, cognitive remediation and psychotherapy

*Corresponding author: Rachit Patel, Department of Psychiatry, Stamford Hospital Stamford, CT 06904, USA, Tel: +1 2032767111; E-mail: rachithpatel@gmail.com

Received September 02, 2015; Accepted November 18, 2015; Published November 28, 2015

Citation: Patel R, Stuart KM, Nambudiri D (2015) Improved Treatment Acceptance and Adherence Following the Diagnosis of Multiple Malformations of Cortical Development in a Patient with Psychosis. Brain Disord Ther 4:192. doi:10.4172/2168-975X.1000192

Copyright: @ 2015 Patel R, et al. This is an open-access article distributed under the terms of the Creative Commons Attribution License, which permits unrestricted use, distribution, and reproduction in any medium, provided the original author and source are credited. 
have been proposed as adjuvant treatments to increase compliance and to decrease the rate of relapse. Most of these interventions have been shown to increase compliance and to decrease the rate of relapse, but the most robust results have been achieved with cognitive behavioral therapy [6]. There is evidence supporting the efficacy of CBT in the treatment of schizophrenia. Turkington et al summarize the main techniques as follows: develop a therapeutic alliance based on the patient's perspective, develop alternative explanations of schizophrenia symptoms, reduce the impact of positive and negative symptoms, offer alternatives to the medical model to address medication adherence [7]. In this case, even with a good therapeutic alliance, the patient was reluctant to accept treatment. However, the therapeutic alliance opened the door to the patient agreeing to further investigational studies. The discovery of an organic basis for his psychosis on MRI provided him with an alternative explanation for his symptomatology in lieu of having to accept that he had a mental disorder. With these interventions, the patient was more amenable to accepting and adhering to antipsychotic treatment that in turn would help improve his insight. In the longer term, the patient demonstrated an improvement in the positive symptoms of his illness with a concurrent improvement in his overall functioning.

\section{Conclusion}

This case demonstrates improved treatment acceptance and adherence in a patient with psychiatric symptoms following the appropriate diagnosis of a neurological disorder with the use of neuroimaging. Neuroimaging findings can be incorporated into a cognitive behavioral therapy model that can be used as an adjunctive treatment for psychotic illnesses. Further advancements in our ability to diagnose potential organic etiologies of neuropsychiatric symptoms may lead to improved treatment outcomes. Furthermore, this case adds to the literature by describing the first instance of psychotic symptoms occurring in the setting of both bilateral periventricular heterotopia and focal cortical dysplasia.

\section{References}

1. Leventer RJ, Guerrini R, Dobyns WB (2008) Malformations of cortical development and epilepsy. Dialogues Clin Neurosci 10: 47-62.

2. Fry AE, Kerr MP, Gibbon F, Turnpenny PD, Hamandi K, et al. (2013) Neuropsychiatric disease in patients with periventricular heterotopia. J Neuropsychiatry Clin Neurosci 25: 26-31.

3. Nopoulos P, Swayze V, Flaum M, Andreasen NC (1998) Incidence of ectopic gray matter in patients with schizophrenia and healthy control subjects studied with MRI. J Neuropsychiatry Clin Neurosci 10: 351-353.

4. Bourgeois JA, Nisenbaum J, Drexler KG, Dobbins KM, Hall MJ (1992) A case of subcortical grey matter heterotopia presenting as bipolar disorder. Compr Psychiatry 33: 407-410.

5. Zygmunt A, Olfson M, Boyer CA, Mechanic D (2002) Interventions to improve medication adherence in schizophrenia. Am J Psychiatry 159: 1653-1664.

6. Saba, Ghassen, et al. (2007) Patients' health literacy in psychotic disorders Neuropsychiatr Dis Treat 3: 511-517.

7. Turkington D, Kingdon D, Weiden PJ (2006) Cognitive-Behavioral Therapy for Schizophrenia: A Review. Am J Psychiatry 163: 365-373. 